\title{
THE EFFECT OF FREEZING AND MELTING ON THE EFFICIENCY OF LATENT HEAT STORAGE SYSTEMS
}

\author{
SALVADOR ACEVES-SABORIO, HAJME NAKAMURA \\ AND GORDON M. REISTAD \\ Department of Mechanical Engineering, Daido Institute of \\ Technology, Nagoya 457
}

Key Words: Energy, Heat Transfer, Thermodynamics, Latent Heat Storage, Freezing and Melting, Exergy

\begin{abstract}
This paper presents a thermodynamic analysis of latent heat storage systems (LHSSs). The study is based on a model that, although simplified, takes full account of the physics of the phase-change process that takes place in the LHSS during charge and discharge. The model characterizes an LHSS with only two parameters and four operating temperatures. This simplicity allows a detailed study of the unit. Two different situations are analyzed. The first is an LHSS in which the phase-change material (PCM) is stored in a single container. The second is an LHSS with many small cells containing the phase-change material.

The simplified model presented in this paper is successful in describing the effects of the physics of the phasechange process on the performance of LHSSs. Optimum efficiencies and phase change temperatures in the LHSSs related to the inlet temperature of the charge fluid are obtained based on exergy analysis. The results also indicate that the insulating effect of the solid layer during the discharge process reduces both the exergetic efficiency and the storage capacity of the unit. On the other hand, the study points to enhanced heat transfer inside the unit as very important for improving LHSSs performance.
\end{abstract}

\section{Introduction}

Over the last few years there has been substantial interest in latent heat storage systems (LHSSs) due to the desirability of obtaining a high energy storage density and a high exergetic efficiency while operating within a narrow temperature range. The recent literature shows some studies $^{1,2,5)}$ focusing on second-law analysis of LHSSs. The main results of these studies are in agreement with the expectations that LHSSs can operate at sufficiently high efficiencies and storage densities to make them attractive. These studies have been carried out to various degrees of detail but they neglect the physics of the phase-change process in both the charge and the discharge processes and consider a constant and uniform overall heat transfer coefficient between the phase-change material (PCM) and the charge and discharge fluids, with the exception of De Lucia and Bejan ${ }^{6,7)}$, who consider the effect of the phase-change process for a restricted set of conditions.

On the other hand, overall system analyses ${ }^{11,13)}$, which consider the interactions of the LHSS with other system components (i.e. solar collectors, loads), indicate that the physics of the phase-change process (especially the insulating effect of the solid PCM) plays an important role in reducing the efficiency of LHSSs, limiting their applicability in many circumstances.

The aim of this paper is to develop a model of a LHSS that, although simplified, takes full account of the

\footnotetext{
* Received May 26, 1994. Correspondence concerning this article should be addressed to $\mathrm{H}$. Nakamura.

**S. Aceves-Saborio is currently at Lawrence-Livermore Nat. Lab., Livermore, CA94551. Gordon M. Reistad is at Dept. of Mech. Engng., Oregon State Univ., Corvallis, OR97331
}

physics of the freezing-melting process. The results show to which extent the phase-change process affects the efficiency of the LHSS. The analysis is done in terms of exergy, since it has been shown (in a previous study ${ }^{5)}$ and many others) that this property best represents the thermodynamic value of various grades of energy.

\section{Model Description}

This paper presents and analyzes two LHSS models. Model I considers an LHSS consisting of a single heat storage container. Model II considers the LHSS to be made of many small heat storage cells. Although both of these models are highly simplified, they include physical descriptions of the phase change process inside the LHSS. Figure 1 shows a schematic of the LHSS configuration for both models. The arrows in the figure indicate the charge and discharge fluid flow direction. Charge and discharge fluids are considered to flow in the same direction because it is found that this configuration (parallel flow) results in a higher LHSS efficiency for Model II.

Figure 1 shows that the PCM in both models is heated from below during the charge process. Heating the PCM from below results in natural convection melting (after a brief period of conduction-dominated melting ${ }^{4,9,14)}$ ). Heat transfer research ${ }^{17)}$ has indicated that the heat transfer coefficient during natural convection melting does not change with time, as long as some solid PCM remains. During discharge (freezing), heat transfer is dominated by conduction through the solid phase ${ }^{16)}$.

The main assumptions for both models are as 
(MODEL I)
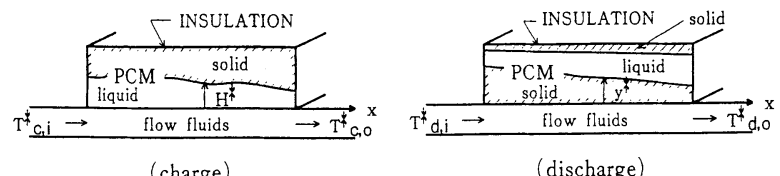

(discharge)

(MODEL II)

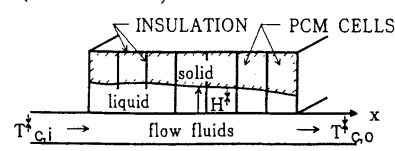

(charge)

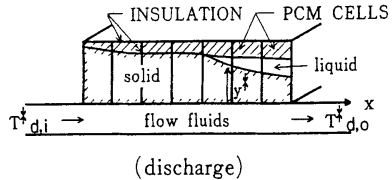

Fig. 1 Schematic of a single storage container LHSS (Model I) and a multiple storage container LHSS (Model II)

follows

1) Sensible energy stored in the PCM is negligible, compared to the latent energy stored (the Stefan number, Ste $=c_{p} *\left(T_{c} *-T_{p} *\right) / L$ is small $)$.

2) The PCM has a well-defined phase change temperature $T_{p}{ }^{*}$. Phase change always occurs at the temperature $T_{p}^{*}$. There is no subcooling in the PCM.

The process starts with the PCM in solid state at the dead state temperature. Suddenly, the charge heat transfer fluid starts flowing into the system. As a consequence, the PCM changes its temperature instantaneously from the dead state temperature to the phase change temperature (due to the neglect of the sensible energy) and starts melting. Before the PCM has melted totally, the charge process ends, and is followed by the discharge process. The heat transfer fluid for discharge circulates through the system until the PCM freezes totally, returning to its initial condition.

\subsection{Model I. Single-storage container}

Model I is a model of an LHSS in which all the PCM is stored in a single container. While this is not the situation most often encountered in typical LHSSs, Model I is studied in this paper to simplify the required analysis. In addition to this, it is shown in this paper that the exergetic efficiencies obtained from Model I represent higher bounds to the efficiencies that can be obtained for Model II, which describes an LHSS with more detail.

As previously described, the LHSS is heated from below during the charge process. For this condition, the following equation has been selected from those recommended in the literature ${ }^{3,12)}$ for Rayleigh numbers higher than $10^{5}$.

$$
N u \equiv h^{*} H^{*} / k_{1}^{*}=0.069 R a^{(1 / 3)} \operatorname{Pr}^{0.074}
$$

with

$$
R a=\left[g^{*} \beta^{*}\left({T_{W}}^{*}-T_{P}^{*}\right) H^{* 3} \operatorname{Pr} / v^{* 2}\right]
$$

In these equations, $T_{w} *$ is the wall temperature, and $H^{*}$ is the height of the liquid cavity (the volume melted from the solid). In this paper, a superscript $*$ indicates a dimensional variable. It is important to notice that the heat transfer coefficient, $h^{*}$, given in Eq. (1), is not a function of $H^{*}$, and therefore, $h^{*}$ during charge does not change as the melting process advances.

This analysis assumes that the main contribution to the thermal resistance between the charge fluid and the PCM is due to the natural convection melting of the PCM. This assumption is equivalent to assuming that $h^{*} \approx U^{*}$, where $U^{*}$ is the overall heat transfer coefficient. The assumption also implies that $T_{w}{ }^{*}$ is approximately equal to the charge fluid temperature, $T_{c}{ }^{*}$, at the axial position being considered. Therefore, $U^{*}$ for charge is proportional to $\left(T_{c}^{*}\right.$ - $\left.T_{p}{ }^{*}\right)^{(1 / 3)}$. Equation (1) was developed for natural convection without melting. However, it has been indicated ${ }^{17)}$ that melting has no effect on natural convection. Therefore, the equation is much more appropriate for this analysis than an assumption of constant $U^{* 1}$.

The differential equation governing the temperature of the charge fluid, written in terms of non-dimensional variables is,

$$
\frac{d T_{c}}{d x}+N T U\left(T_{c}-T_{P}\right)=0
$$

where $x=x * / l *, T=T^{*} / T_{d s}{ }^{*}$, and $N T U=U * A * / m_{c}{ }^{*} c_{p c}{ }^{*}$.

As Eqs. (1) and (2) indicate, the heat transfer coefficient, and therefore $N T U$, is proportional to $\left(T_{c}-T_{p}\right)^{(1 / 3)}$. This fact has to be written explicitly in Eq. (3) before it can be solved. This is done by rewriting Eq. (3) as follows:

$$
\frac{d T_{c}}{d x}+N T U_{n}\left(T_{c}-T_{p}\right)^{(4 / 3)}=0
$$

where a new parameter, $N T U_{n}$, which is not a function of either $x$ or $t$, has been defined as:

$$
N T U_{n}=N T U /\left(T_{c}-T_{p}\right)^{(1 / 3)}
$$

Integration of Eq. (4) results in the following equation for the outlet charge fluid temperature:

$$
T_{c, o}=T_{p}+\left[\left(T_{c, i}-T_{p}\right)^{(-1 / 3)}+N T U_{n} / 3\right]^{-3}
$$

The exergetic efficiency for the charge process (defined as the ratio between the exergy stored by the unit and the exergy input to the unit as a basis of dead state) can be written in terms of non-dimensional temperatures as follows ${ }^{1)}$,

$$
\Phi_{c}=\frac{\left(T_{c, i}-T_{c, o}\right)\left(1-1 / T_{p}\right)}{\left[T_{c, i}-1-\ln \left(T_{c, i}\right)\right]}
$$

The exergetic efficiency is an indication of the degree of thermodynamic perfection of any process and therefore the LHSS is considered optimum when a maximum exergetic efficiency is obtained. Substituting Eq. (6) into (7), it is straightforward to find the optimum phasechange temperature for charge, $T_{p}=T_{p \text {, opt }}$, for which $d \Phi_{c} / d T_{p}=0$.

In the discharge process, heat transfer occurs mainly due to conduction through the solid phase. The following three non-dimensional equations can be written for the rate 
of overall energy discharged from the PCM,

$$
\begin{aligned}
& \frac{d Q_{d}}{d x}=\frac{\partial T_{d}(x, t)}{\partial x} \\
& \frac{d Q_{d}}{d x}=k \frac{T_{p}-T_{d}(x, t)}{y(x, t)} \\
& \frac{d Q_{d}}{d x}=\frac{\partial y(x, t)}{\partial t}
\end{aligned}
$$

where the non-dimensional variables are defined as follows:

$$
\begin{aligned}
& Q=Q^{*} /\left(m_{c} * c_{p c} * t_{c} * T_{d s}{ }^{*}\right) \\
& y=y^{*} L^{*} \rho^{*} A^{*} /\left(m_{c}{ }^{*} c_{p c}{ }^{*} t_{c} * T_{d s} *\right) \\
& k=k_{s}{ }^{*} A^{* 2} L^{*} \rho^{*} /\left(m_{d} * c_{p d}{ }^{*} m_{c}{ }^{*} c_{p c}{ }^{*} t_{c} * T_{d s}{ }^{*}\right) \\
& t=m_{d}{ }^{*} c_{p d}{ }^{*} /\left(m_{c}{ }^{*} c_{p c}{ }^{*} t_{c}{ }^{*}\right)
\end{aligned}
$$

It is worth noticing that the present formulation specifies completely the LHSS with only two parameters and a set of operating temperatures. The two parameters are, $N T U_{n}$ for charge and $k$ for discharge. The parameter $k$ is equivalent to $N T U_{n}$ for discharge, since it indicates the size and the discharge heat transfer capability of the unit. In addition to this, it can be seen from Eqs.(6) and (11) that the nondimensional energy stored at the end of the charge process, $Q$, is not a function of time. System performance is also independent of the overall PCM thickness as long as the thickness is large enough so that the PCM does not melt totally during charge.

The system of Eqs. (8), (9) and (10) is solved to obtain $T_{d, o}(t)$, the discharge fluid outlet temperature, as a function of time. The equations are solved numerically. The procedure is straightforward, and is therefore omitted. The calculations are carried out for $T_{d, i}=1$, and $N T U_{n}=5$, considered to be representative values. A range of values is used for $k$ since there is more uncertainty on the values that this variable may have for typical LHSSs. An experimental unit ${ }^{8)}$ was built with $k \approx 0.025$. Based on this value, the range $0.01<k<0.2$ is selected.

The analysis for Model I assumes that some liquid remains at all longitudinal positions along the LHSS until the moment when the PCM freezes completely, so that all the heat transfer area remains "active" during the discharge process. This assumption is expected to be accurate only for small values of $k$. Model II, described later in this paper, takes into account that some parts of the LHSS freeze completely before others and considers that cells that freeze completely do not transfer energy. Therefore, Model II is expected to describe better the performance of LHSSs. Model I is presented to simplify the calculations, and because the results for Model I have a value as higher bounds to the efficiencies obtained for Model II.

The values of $T_{d, o}(t)$ obtained from the analysis are used to calculate the exergetic efficiency for the overall charge-discharge process. The overall exergetic efficiency, $\Phi$, is given as the ratio between the total exergy

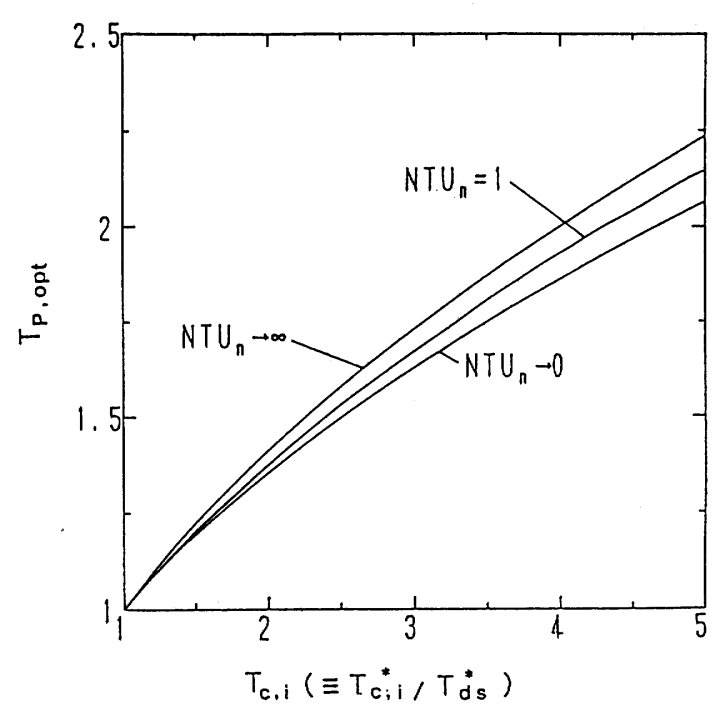

Fig. 2 Optimum phase-change temperature for the charge process of Model I as a function of $T_{c, i}$ with $N T U_{n}$ as a parameter

flowing out with the discharge fluid (integrated over time, since $T_{d, o}$ is a function of time), and the total exergy input to the unit, and is given by the following expression:

$$
\Phi=\frac{\int_{0}^{t_{d}}\left[T_{d, o}-1-\ln \left(T_{d, o}\right)\right] d t}{\left[T_{c, i}-1-\ln \left(T_{c, i}\right)\right]}
$$

where $t_{d}$ is the non-dimensional time required to complete the discharge process (to freeze the PCM completely). The expression for $\Phi$ is then optimized by finding the $T_{p}$ that results in a maximum exergetic efficiency for each given $T_{c, i}, k$ and $N T U_{n}$. The procedure consists of solving $\mathrm{d} \Phi / d T_{p}$ $=0$. The calculation has to be done numerically since $\Phi$ is a function of $T_{d, o}$ which is obtained numerically from Eqs. (8), (9), and (10).

\subsection{Model II. Multiple cell storage unit}

Model II represents an LHSS in which the PCM is stored in many individual cells. This model is expected to describe the conditions existing in actual LHSSs more accurately than Model I. The cells exchange heat with the charge and discharge fluids, but are insulated from each other. Figure 1 shows a schematic of this unit, which is identical to that of Model I except for the large number of insulated cells.

It can be readily seen that the charge process is not affected by insulation between the cells (assuming that the space occupied by the insulation is small), and therefore the results obtained for Model I in the charge process apply identically for Model II, assuming that the Nusselt number is still proportional to $R a^{1 / 3}$. For the discharge process, however, the situation is different, because each cell stores a certain amount of thermal energy during the charge process and this cannot be shared with the neighboring cells. Therefore, some cells are depleted of their energy inventory before others, the order of depletion depending on the values of $N T U_{n}$ and $k$. Depletion of the cells reduces 


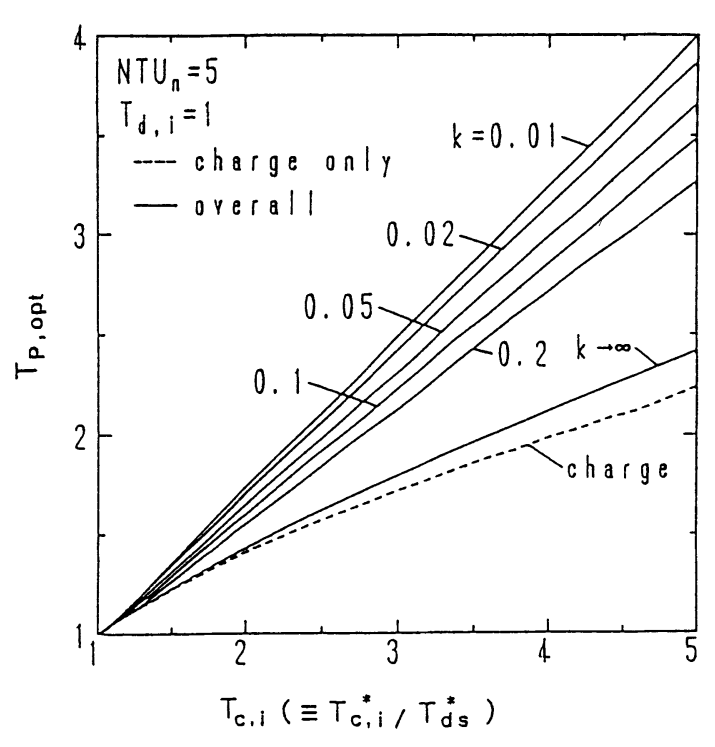

Fig. 3 Optimum phase change temperatures for the overall charge-discharge process, as a function of $T_{c, \text {, }}$, for $N T U_{n}=5, \mathrm{~T}_{\mathrm{d}, \mathrm{i}}=1$, and for different values of $k$

heat transfer rates (a depleted cell does not transfer energy). This reduces the temperature of the outlet discharge fluid, therefore reducing the outlet exergy with respect to the value obtained for Model I. The efficiency of Model II can be as high as the efficiency of Model I only in the case when all the storage cells are depleted of their energy at exactly the same time.

\section{Results and Discussion}

\subsection{Model I}

Figure 2 shows the optimum phase change temperature for the charge process. This figure shows $T_{p \text {, opt }}$ to vary as a function of $N T U_{n}$ within a small range. The range is bounded by the following expressions,

$$
T_{p, o p t}=\left(T_{c, i}\right)^{0.5}, \text { for } N T U_{n} \rightarrow \infty
$$

and

$$
T_{p, o p t}=\left[1+\left(1+48 T_{c, i}\right)^{0.5}\right] / 8, \text { for } N T U_{n} \rightarrow 0
$$

The asymptotic expressions in Eqs. (13) and (14) indicate respectively optimum phase change temperatures for very large and very small LHSSs. While these equations may not apply to most practical LHSSs, they are still important, because they indicate bounds for the possible $T_{p, o p t}$ values $\left(T_{p, o p t}\right.$ for any $T_{c, i}$ cannot fall outside the area bounded by these lines). Other results in this paper also use this type of asymptotic expansion to express bounds in LHSS behavior for very large or very small LHSSs.

It is interesting to see that the result presented in Eq. (13) has been obtained in the past as the value of $T_{p, o p t}$ for the charge process in an LHSS with a constant and uniform heat transfer coefficient ${ }^{5)}$.

Figure 3 shows the phase change temperature that

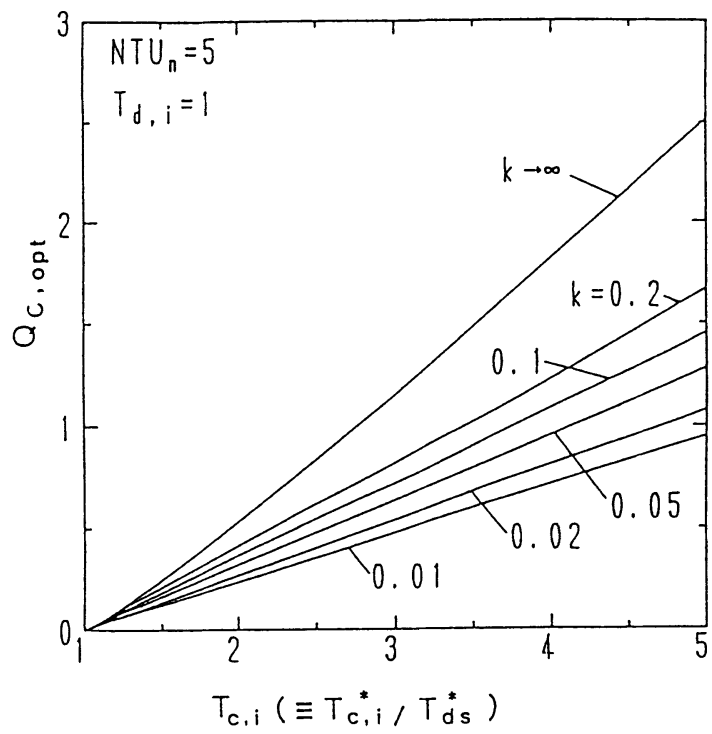

Fig. 4 Overall thermal energy stored in the latent heat storage unit at the optimum operating point $\left(T_{p}=T_{p . o p t}\right)$ as a function of $T_{c, i}$, for $N T U_{n}=5, T_{d, i}=1$ and for different values of $k$

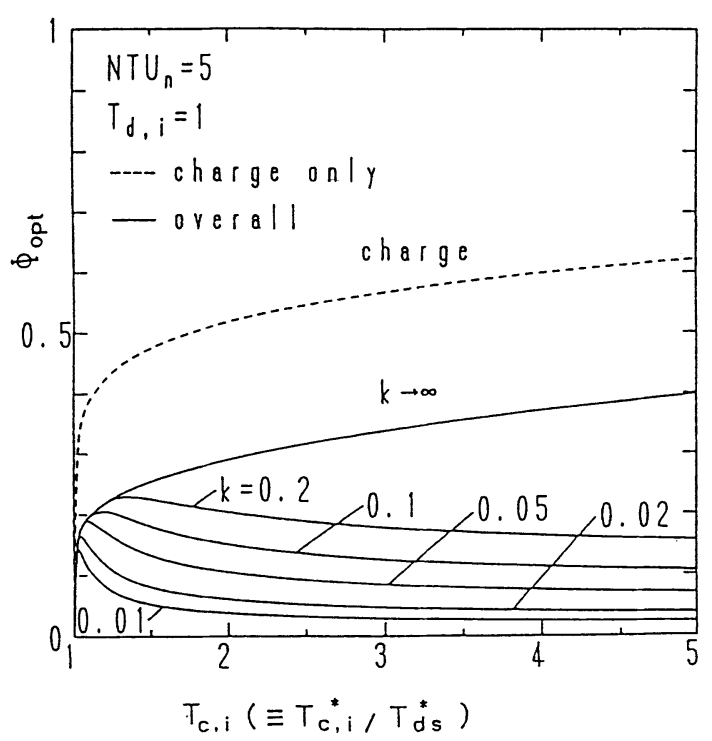

Fig. 5 Optimum overall exergetic efficiency, as a function of $T_{k}, i$, for $N T U_{n}=5, T_{d, i}=1$ and for different values of

results in optimum overall exergetic efficiency, $T_{p, o p t}$, as a function of $T_{c, i}$ for different values of $k$. The optimum phase change temperature for the charge process is also shown for comparison. It can be seen that the lines obtained for finite values of $\mathrm{k}$ spread very much apart from the charge optimum temperature. The lines for finite values of $\mathrm{k}$ are almost straight and have a large slope, greater than 0.75 for small values of $k$.

Figure 4 shows the energy, $Q_{c, \text { opt }}$, stored in the LHSS, for $T_{p}=T_{p, o p t}$, as a function of $T_{c, i}$, for different values of $k$. The energy stored increases linearly as a function of $T_{c, i}$ with the slopes of the lines being a strong 


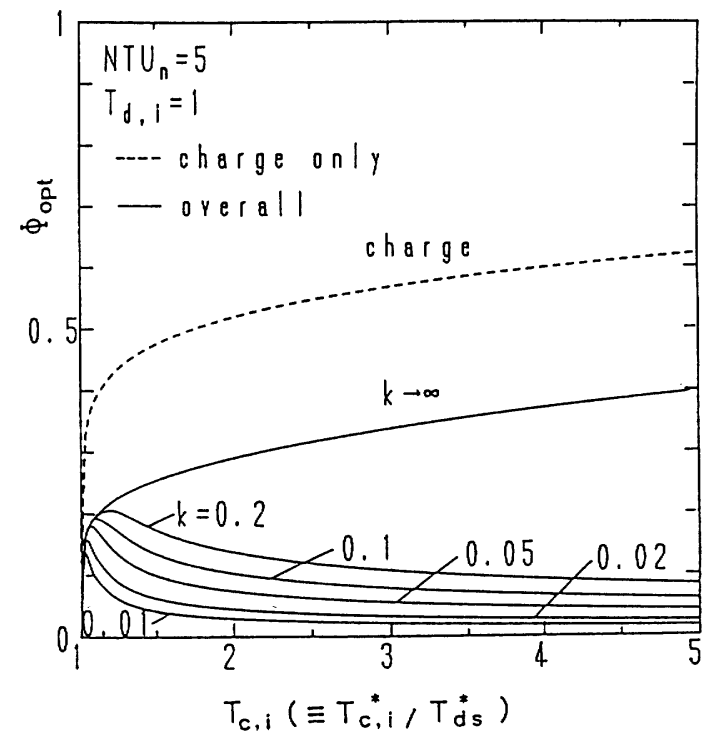

Fig. 6 Optimum overall exergetic efficiency for a unit consisting of multiple storage capsules in parallel flow configuration (Model II) as a function of $T_{c, i}$, for $N T U_{n}=5, T_{d, i}=1$ and for different values of $k$

function of $k$.

Figure 5 shows the optimum overall exergetic efficiency, $\Phi_{o p t}$, as a function of $T_{c, i}$, for different values of $k$. The figure shows optimum exergetic efficiencies for finite $k$ reaching a narrow peak for a $T_{c, i}=T_{c, i, o p t}$ only slightly greater than 1, before dropping to approach asymptotic values for large $T_{c, i}$. The shape of the efficiency curves is the result of two competing effects. As $T_{c, i}$ increases, the driving potential for the heat transfer process (the temperature difference) increases. This tends to increase the efficiency of the LHSS. However, increasing $T_{c, i}$ also increases the amount of energy stored in the LHSS (Figure 4). This causes the solid layer thickness to increase, increasing the heat transfer resistance, which tends to decrease the efficiency.

The previous paragraph has shown that the insulating effect of the solid layer causes a decrease in efficiency with an increased $T_{c, i}$. The insulating effect of the solid layer has another important effect that is also detrimental to LHSS performance. This effect is a reduction of the storage capacity of the unit. As previously shown, $T_{c, i, \text { opt }}$ is low and the energy storage capacity of the unit increases linearly with $T_{c, i}$. Therefore, operating at maximum efficiency, for which $T_{c, i}=T_{c, i, o p t}$, results in a low storage capacity. The storage capacity can be increased by increasing $T_{c, i}$ to values higher than $T_{c, i, o p t}$, but only at the cost of reducing the efficiency of the unit.

The drops in efficiency and storage capacity caused by an increase in $T_{c, i}$ are due to the insulating effect of the solid phase during discharge and therefore these effects do not appear in studies that consider a constant overall heat transfer coefficient ${ }^{1,2,5)}$. However, the drop in efficiency has been recognized to be important in earlier studies that consider the LHSS as a part of an overall system ${ }^{11,13)}$. On the other hand, Figure 5 also shows the large possibilities for improving the efficiency of the system by increasing the value of $k$. Therefore, these results indicate that research on enhanced heat transfer in $\operatorname{LHSSs}^{10,15)}$ is very important for the practical applicability of these systems.

\subsection{Model II}

Figure 6 shows the optimum overall exergetic efficiency for Model II. The curves for exergetic efficiency behave in a similar way to those obtained for Model I (Fig. 5) but the efficiencies and the values for $T_{c \text {, in opt }}$ are notoriously lower for Model II. As previously explained, the drop in efficiency occurs because the energy in the storage cells is not depleted simultaneously.

Figures 5 and 6 indicate that the efficiency for Model $\mathrm{I}$ is a higher bound for the efficiency of the more detailed Model II.

\section{Conclusions}

This paper has presented two models of an LHSS. The models, although simplified, take full account of the physics of the phase-change processes that take place during LHSS charge and discharge. The model describes LHSS operation with only two parameters and four operation temperatures, therefore allowing a detailed study of how the operating conditions affect the efficiency of the unit.

The models presented in this paper, although very simplified, are successful in evaluating the insulating effect of the solid layer that forms during the discharge process. The paper shows that the solid layer causes the efficiency and the storage capacity of the unit to drop, in agreement with what has been indicated by detailed system analyses. An increase in storage capacity can only be obtained at the cost of reducirig the exergetic efficiency of the unit. On the other hand, the results also indicate the substantial advantages that can be obtained by operating LHSSs with large discharge heat transfer parameters, and therefore point to research on enhanced heat transfer with freezing as very important for the practical applicability of LHSSs.

\section{Acknowledgements}

This work was financially supported by the JSPS Postdoctoral Fellowship for Foreign Researchers and the Monbusho Grant-in-Aid for Encouragement of Young Scientists.

One of the authors (S.A.) wishes to acknowledge the financial support received from the U.S. National Science Foundation and the Japan Society for the Promotion of Science.

\begin{tabular}{llr}
\multicolumn{2}{l}{ Nomenclature } \\
$A$ & $=$ area & {$\left[\mathrm{m}^{2}\right]$} \\
$c_{p}$ & $=$ specific heat & {$[\mathrm{J} /(\mathrm{g} \cdot \mathrm{K})]$} \\
$g$ & $=$ acceleration of gravity & {$\left[\mathrm{m} / \mathrm{s}^{2}\right]$} \\
$h$ & $=$ heat transfer coefficient & {$\left[\mathrm{W} / \mathrm{m}^{2} \cdot \mathrm{K}\right]$} \\
$H^{*}$ & $=$ height of the liquid cavity & {$[\mathrm{m}]$} \\
$k_{l}$ & $=$ liquid thermal conductivity & {$[\mathrm{W} / \mathrm{m} \cdot \mathrm{K}]$} \\
$k_{s}$ & $=$ solid thermal conductivity & {$[\mathrm{W} / \mathrm{m} \cdot \mathrm{K}]$} \\
$k$ & $=$ non-dimensional discharge parameter & {$[-]$} \\
$L$ & $=$ latent heat of solidification & {$[\mathrm{J} / \mathrm{g}]$} \\
$l$ & $=$ length & {$[\mathrm{m}]$} \\
$m$ & $=$ mass flow rate & {$[\mathrm{g} / \mathrm{s}]$}
\end{tabular}




\begin{tabular}{|c|c|c|c|}
\hline$N T U$ & $=$ & number of heat transfer units & {$[-]$} \\
\hline$N T U_{n}$ & $=$ & modified NTU parameter(Eq.(5)) & {$[-]$} \\
\hline $\mathrm{Nu}$ & $=$ & Nusselt number & {$[-]$} \\
\hline $\operatorname{Pr}$ & $=$ & Prandtl number & {$[-]$} \\
\hline$Q$ & $=$ & non-dimensional overall heat transfer & {$[-]$} \\
\hline$R a$ & $=$ & Rayleigh number & {$[-]$} \\
\hline$t$ & $=$ & non-dimensional time length of the process & {$[-]$} \\
\hline$T$ & $=$ & non-dimensional temperature & {$[-]$} \\
\hline$U^{*}$ & $=$ & overall heat transfer coefficient & {$\left[\mathrm{W} / \mathrm{m}^{2} \cdot \mathrm{K}\right]$} \\
\hline$x$ & $=$ & non-dimensional axial coordinate & {$[-]$} \\
\hline$y$ & $=$ & non-dimensional solid layer thickness & {$[-]$} \\
\hline$\beta^{*}$ & $=$ & coefficient of thermal expansion & {$[1 / \mathrm{K}]$} \\
\hline$v^{*}$ & $=$ & kinematic viscosity & {$\left[\mathrm{m}^{2} / \mathrm{s}\right]$} \\
\hline$\Phi *$ & $=$ & exergetic efficiency & {$[-]$} \\
\hline$\rho^{*}$ & $=$ & density & {$\left[\mathrm{kg} / \mathrm{m}^{3}\right]$} \\
\hline
\end{tabular}

$<$ Superscripts $>$

$\begin{array}{ll}* & =\text { dimensional variable } \\ <\text { Subscripts } & \\ \mathrm{c} & =\text { charge process } \\ \mathrm{d} & =\text { discharge process } \\ \mathrm{ds} & =\text { dead state condition } \\ \mathrm{i} & =\text { inlet } \\ \mathrm{o} & =\text { outlet } \\ \mathrm{opt} & =\text { optimum } \\ \mathrm{p} & =\text { phase change condition } \\ \mathrm{w} & =\text { wall }\end{array}$

\section{Literature Cited}

1) Aceves-Saborio, S.M., H. Nakamura, and G. M. Reistad: J. Energy Resources Technology, 116, 79-86 (1994)

2) Adebiyi, G.A. and L.D. Russell: ASME HTD-Vol.80, 9-20 (1987)

3) Bejan, A.: Convection Heat Transfer, John Wiley and Sons (1984)

4) Benard, C., D. Gobin, and F. Martinez: J. Heat Transfer, 107, 794803 (1985)

5) Bjurstrom, H. and B. Carlsson: Heat Recovery Systems, 5, 233-250 (1985)

6) De Lucia, M. and A. Bejan: J. Solar Energy Engineering, 112, 110116 (1990)

7) De Lucia, M. and A. Bejan: J. Solar Energy Engineering, 113, 2-10 (1991)

8) Farid, M.M. and A. Kanzawa:J. Solar Energy Engineering, 111, 152 157 (1989)

9) Hale, N.W. and R. Viskanta: Int. J. Heat and Mass Transfer, 23, 283292 (1980)

10) Henze, R.H. and J.A.C. Humphrey: Int. J. Heat and Mass Transfer, 24, 459-474, (1981)

11) Marshall, R.: Solar Energy, 32, 41-48 (1984)

12) McAdams, W.H.: Heat Transmission (3rd ed.), McGraw-Hill (1954)

13) Morrison, D.J., and S.I. Abdel-Khalik: Solar Energy, 20, 57-67 (1978)

14) Okada, M.: Int. J. Heat and Mass Transfer, 27, 2057-2066(1984)

15) Smith, R.N., T.E. Ebersole, and F.P. Griffin: J. Solar Energy Engineering, 102, 112-118 (1980)

16) Sparrow, E.M. and J.A. Broadbent: J. Heat Transfer, 105, 217-225 (1983).

17) Viskanta, R.: in Kakac, Aung, and Viskanta, eds.: "Natural Convection, Fundamentals and Applications," Hemisphere Publishing Corporation, (1985) 